\title{
Pentraxin-3, Angiopoietin-Like Protein-3, Angiopoietin-Like Protein-4 and Angiopoietin-Like Protein-8 Levels in Morbid Obese Children
}

Mustafa Metin Donma ${ }^{1 *}$, Muhammet Demirkol $^{2}$, Hakan Ekmekci $^{3}$, Ozlem Balci Ekmekci ${ }^{3}$, Orkide Donma ${ }^{3}$

\footnotetext{
${ }^{1}$ Prof. Dr., Namik Kemal University, Medical Faculty, Department of Pediatrics, Tekirdag, Turkey;

${ }^{2} \mathrm{Sp}$. Dr., Ministry of Health, Cerkezkoy State Hospital, Pediatrics Clinics, Tekirdag, Turkey;

${ }^{3}$ Prof. Dr. Istanbul University, Cerrahpasa Medical Faculty, Department of Medical Biochemistry, Cerrahpasa, Istanbul, Turkey.
}

Correspondence: Prof. Dr. Mustafa Metin Donma, E-mail addresses: mdonma@gmail.com, mdonma@nku.edu.tr, Telephone no: 00-90-53237172 07, Fax no: 00-90-282-250 9928.

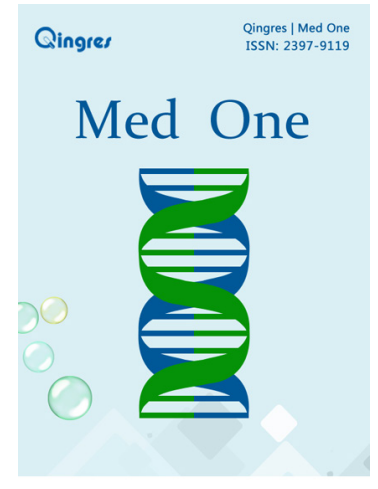

http://mo.qingres.com

\section{GOPEN ACCESS}

DOI: $10.20900 / \mathrm{mo} .20170029$

Received: October 18, 2017

Accepted: November 30, 2017

Published: December 22, 2017

Copyright: (2017 Cain et al. This is an open access article distributed under the terms of the Creative Commons Attribution License, which permits unrestricted use, distribution, and reproduction in any medium, provided the original author and source are credited.

\begin{abstract}
A low-grade inflammatory state accompanies obesity and metabolic syndrome (MetS), which are risk factors for cardiovascular diseases (CVD) in children's future lives. The aim of this study is to investigate possible associations of pentraxin 3 (PTX3), a promising new marker for CVDs, with angiopoietin-like proteins (ANGPTL) in morbid obese (MO) children and their potential uses in the prediagnosis of MetS. Thirty normal weight (NW) and $50 \mathrm{MO}$ prepubertal children without MetS symptoms, a total of 80 , participated in the study. Using percentile tables for age and gender recommended by WHO, children whose percentiles were between 15-85 and above 99 were included in NW and MO groups, respectively. Anthropometric measurements, $\mathrm{BMI}$ and HOMA-IR values were recorded. Serum PTX3, ANGPTL3, ANGPTL4, ANGPTL8/betatrophin levels were determined by enzymelinked immunosorbent assay (ELISA). Statistical analyses were performed by SPSS. $p<0.05$ was the degree of statistical significance. There were no differences between PTX3, ANGPTL3 and ANGPTL4 levels of groups. ANGPTL8/betatrophin levels were significantly higher in MO children $(p \leq 0.001)$. A significant correlation was observed between PTX3 and ANGPTL4 $(r=0.297, p \leq 0.05)$ in the same group. When adjusted for MetS parameters including HDL-Chol, correlations
\end{abstract}


between ANGPTL3-ANGPTL8/betatrophin ( $r=$ $0.327, p \leq 0.05)$ and PTX3-ANGPTL4 $(r=0.337, p \leq$ 0.05 ) were detected. When HDL-Chol was replaced with TRG these associations were disappeared. This study is the first to perform PTX3, ANGPTL3, ANGPTL4, ANGPTL8/betatrophin measurements in prepubertal NW and MO children. Upon evaluation of dyslipidemia in MetS, adjustment for MetS parameters pointed out HDL-Chol as the more valuable parameter than TRG, based upon the existence of ANGPTL3-ANGPTL8/betatrophin as well as PTX3-ANGPTL4 associations.

Keywords: Angiopoietin-like protein 3; Angiopoietinlike protein 4; Angiopoietin-like protein 8; Betatrophin; Childhood obesity; Pentraxin-3

\section{INTRODUCTION}

Obesity is a chronic clinical state associated with low-grade inflammation ${ }^{[1]}$. Many proinflammatory factors including $\mathrm{C}$-reactive protein (CRP) contribute to the process. In the same family, PTX3, being detected mainly in atherosclerotic lesions, is suggested as a promising new marker for inflammatory cardiovascular diseases (CVD) such as atherosclerosis, acute coronary syndrome, chronic heart failure as well as MetS ${ }^{[2-9]}$.

ANGPTL are structurally similar molecules to angiopoietins. Aside from their angiogenic effects, they also participate in several physiologic and pathologic processes. They have specific roles in lipid, carbohydrate, energy metabolisms, hematopoiesis, cancer and inflammation ${ }^{[10-12]}$.

The potential roles of ANGPTL at the crossroads of lipoprotein, fatty acid and glucose metabolisms make these molecules important for the cardiovascular risk ${ }^{[13]}$. Particularly, ANGPTL3, ANGPTL4 and ANGPTL8/betatrophin are involved in the regulation of lipid and lipoprotein metabolisms and provide new hope for MetS ${ }^{[10-17]}$.

ANGPTL4 represses the foam cell formation and may be protective against lipid accumulation ${ }^{[18]}$. ANGPTL3, by way of its effect on insulin, may lead to decreases in ANGPTL4 ${ }^{[10]}$. ANGPTL8/betatrophin contributes to the inhibition of lipoprotein lipase (LPL) by participating in the cleavage of ANGPTL3 and thus, activating it ${ }^{[16,17]}$. ANGPTL8/betatrophin act together with ANGPTL3 to coordinate the trafficking of triacylglycerols (TRG) to tissues ${ }^{[19,20]}$.

Atherosclerosis is the most common cause of CVD, such as myocardial infarction and stroke. Inflammatory mechanisms are known to play a central role in the pathogenesis and progression of atherosclerosis. PTX3 is a modulator molecule of complement system, inflammatory response, angiogenesis, and vascular remodeling, which are critical determinants of CVD. Compared to most inflammatory markers PTX3 displays greater association with coronary artery disease. PTX3 may play an important role in maintaining plaque stability by mediating phagocytosis of late apoptotic macrophages. PTX3 affects lipid metabolism in human macrophages through increasing the uptake of oxidized low-density lipoprotein cholesterol and inhibiting cholesterol efflux pathways such as peroxisome proliferator-activated receptor- $\gamma^{[21,22]}$.

ANGPTL-3, 4 and 8 act as important regulators of plasma lipoprotein levels by inhibiting LPL. ANGPTL4 plays a central role in lipid metabolism and pathophysiology of atherosclerosis. ANGPTL4 can destroy the endothelium and may lead to the initiation of atherosclerosis. The tagged single nucleotide polymorphisms and high serum ANGPTL4 levels are associated with large artery atherosclerotic stroke. MicroRNA-134 accelerates atherogenesis by promoting lipid accumulation and proinflammatory cytokine secretion via the ANGPTL4/LPL pathway. ANGPTL3 cooperates with ANGPTL8 to inhibit LPL. Inactivation of ANGPTL3 reduces plasma TRG and suppresses atherosclerosis. In human clinical trials, antisense oligonucleotide and monoclonal antibodybased inactivation of ANGPTL3 for the treatment of dyslipidaemia and atherosclerosis are being investigated. Oligonucleotides targeting ANGPTL3 retarded the atherosclerosis progression and reduced atherogenic lipoprotein levels ${ }^{[23,27]}$.

Obesity and MetS may be considered as risk factors for CVD. The aim of this study is to determine PTX3 and ANGPTL3, ANGPTL4, ANGPTL8/ betatrophin concentrations in prepubertal NW and MO children for the investigation of their potential uses in the prediagnosis of MO children, who may develop MetS in the future. This study, for the first time, questions if MetS components observed in prepubertal MO children without MetS symptoms may be associated with the signs leading to CVD during their future lives.

\section{MATERIALS AND METHODS}

Thirty NW and fifty MO children without MetS symptoms participated in the study to compare the ANGPTL3, ANGPTL4, ANGPTL8/betatrophin and PTX3 levels in blood serum. All the children were in prepubertal stage (Tanner stage 1). Two groups were matched from the age and female/male ratio points of view. The study protocol was approved by Namik Kemal University Medical Faculty Ethics 
Committee (NKU/TF/GOKAEK/2014/68/10/03-86). The study was supported by Namik Kemal University Scientific Research Fund Projects Coordination Unit Project No: NKUBAP.00.20.TU.14.01. All procedures were performed in accordance with the accepted international standards and the declaration of Helsinki. Written informed consent forms were signed by the parents of all participant children prior to their involvement in the study. Children were classified as NW and MO (15th-85th and $\geq 99$ th percentiles, respectively) based upon body mass index (BMI)for-age and -gender tables recommended by World Health Organization and approved by Ministry of Health.

The basic anthropometric measurements (weight, height, waist circumference, hip circumference, head circumference and neck circumference) were taken and recorded. BMI values of children were calculated.

Blood samples were drawn after an overnight fasting. Venous blood was stored at $-80^{\circ} \mathrm{C}$ following centrifugation until the analyses were performed. The complete blood counts, routine biochemical tests, $\mathrm{HbA} 1 \mathrm{c}$, lipid profile, glucose and insulin analyses were performed. Insulin resistance (IR) was calculated as Homeostatic Model Assessment for IR (HOMA-IR) index using the following formula: [insulin $(\mu \mathrm{lU} / \mathrm{ml})$ * fasting blood glucose $(\mathrm{mg} / \mathrm{dl})] /$ [22.5/0.0555] .

Serum concentrations of the parameters were determined using human PTX3, human ANGPTL3, human ANGPTL4, human ANGPTL8/betatrophin (total) ELISA kits (AVISCERA BIOSCIENCE, Inc. Santa Clara, CA, USA). Inter- and intra-assay coefficient of variation were $8-12 \%$ and $4-6 \%$ for PTX3, 8-12 \% and 4-8 \% for ANGPTL3, 8-10\% and $4-6 \%$ for ANGPTL4, 8-10 $\%$ and $4-6 \%$ for ANGPTL8/betatrophin.

SPSS Version 20 was used for statistical analyses. Data were expressed in mean $\pm S D$. Kolmogorov-Smirnov and Shapiro Wilk tests were used to test the normality for the distribution of the data. The differences between the groups were determined by Student's t-test or Mann-Whitney U test based upon the parametric or non-parametric distribution. The correlation analyses were performed using bivariate and partial correlation tests. $p$ values $<0.05$ were accepted as statistically significant.

\section{RESULTS}

This study was performed on eighty prepubertal children aged between 5.8-9.1 years. The study population was divided into two groups; NW healthy children (Group 1, $\mathrm{n}=30$ ) and MO children without MetS (Group 2, $n=50$ ). No statistically significant differences were noted between ages as well as female/male ratios of Group $1(\mathrm{~F} / \mathrm{M}=1.1$, age $=7.5$ \pm 1.0 years $)$ and Group $2(\mathrm{~F} / \mathrm{M}=1.1$, age $=7.6 \pm 0.9$ years) $(p \geq 0.05)$.

Anthropometric measurements of children in groups were summarized in Table 1.

Table 1. Body mass index, waist circumference, hip circumference, head circumference and neck circumference values of children (mean \pm SD)

\begin{tabular}{lccc}
\hline Parameter & Group 1 (NW) & Group 2 (MO) & P-value \\
\hline BMI (kg/m2) & $15.2 \pm 0.8$ & $25.1 \pm 3.1$ & $\leq 0.001$ \\
Waist circumference $(\mathrm{cm})$ & $53.0 \pm 3.8$ & $79.7 \pm 8.1$ & $\leq 0.001$ \\
Hip circumference $(\mathrm{cm})$ & $59.5 \pm 4.3$ & $84.5 \pm 7.7$ & $\leq 0.001$ \\
Head circumference $(\mathrm{cm})$ & $49.7 \pm 1.6$ & $52.0 \pm 2.5$ & $\leq 0.001$ \\
Neck circumference $(\mathrm{cm})$ & $25.4 \pm 1.3$ & $30.4 \pm 2.9$ & $\leq 0.001$ \\
Waist-to-Hip ratio & $0.89 \pm 0.04$ & $0.95 \pm 0.06$ & $\leq 0.001$ \\
Head-to-Neck ratio & $1.96 \pm 0.09$ & $1.72 \pm 0.12$ & $\leq 0.001$ \\
\hline
\end{tabular}


Statistically significant increases were found in MO children compared to NW healthy children in terms of their BMI, waist circumference, hip circumference, head circumference, neck circumference values and anthropometric ratios $(p$ $\leq 0.001$ ). Blood pressure values and lipid profile of children were tabulated in Table 2.

Table 2. Systolic pressure (SP), diastolic pressure (DP), total cholesterol (TChol), triacylglycerol (TRG), low density lipoprotein cholesterol (LDL-Chol), high density lipoprotein cholesterol (HDL-Chol) values of children in groups (mean \pm SD)

\begin{tabular}{llll}
\hline Parameter & Group 1 (NW) & Group 2 (MO) & P-value \\
\hline SP $(\mathrm{mm} \mathrm{Hg})$ & $102.6 \pm 8.0$ & $107.5 \pm 9.2$ & $\leq 0.05$ \\
DP $(\mathrm{mm} \mathrm{Hg})$ & $66.7 \pm 5.1$ & $70.2 \pm 9.2$ & $\mathrm{NS}$ \\
TChol $(\mathrm{mg} / \mathrm{dl})$ & $163.2 \pm 29.0$ & $167.8 \pm 27.5$ & $\mathrm{NS}$ \\
TRG $(\mathrm{mg} / \mathrm{dl})$ & $60.1 \pm 20.9$ & $78.1 \pm 35.3$ & $\leq 0.05$ \\
LDL-Chol $(\mathrm{mg} / \mathrm{dl})$ & $98.8 \pm 26.5$ & $101.8 \pm 25.3$ & $\mathrm{NS}$ \\
HDL-Chol $(\mathrm{mg} / \mathrm{dl})$ & $53.8 \pm 11.8$ & $50.5 \pm 7.7$ & $\mathrm{NS}$ \\
\hline
\end{tabular}

In MO group, systolic blood pressure and TRG values were significantly higher than those in NW group $(p \leq 0.05)$. Diastolic blood pressure, Total cholestereol (Tchol), LDL-Chol and HDL-Chol values did not differ between the groups.

Values for parameters related to IR were listed in Table 3.

Table 3. Blood glucose, insulin, HbA1c, HOMA-IR values (mean \pm SD)

\begin{tabular}{llll}
\hline Parameter & Group 1 (NW) & Group 2 (MO) & P-value \\
\hline Glucose $(\mathrm{mg} / \mathrm{dl})$ & $86.1 \pm 8.3$ & $86.2 \pm 5.6$ & $\mathrm{NS}$ \\
Insulin $(\mu \mathrm{U} / \mathrm{l})$ & $3.3 \pm 3.5$ & $9.7 \pm 7.6$ & $\leq 0.001$ \\
HbA1c $(\%)$ & $5.4 \pm 0.8$ & $5.7 \pm 1.7$ & $\mathrm{NS}$ \\
HOMA-IR & $0.73 \pm 0.80$ & $2.11 \pm 1.75$ & $\leq 0.001$ \\
\hline
\end{tabular}

There were no statistically significant differences between groups in terms of fasting blood glucose and glycated hemoglobin levels. Significantly increased insulin and IR index, HOMA-
IR values were observed in MO children $(p \leq 0.001)$.

Findings related to PTX 3 and ANGPTL concentrations of children were summarized in Table 4. 
Table 4. Pentraxin 3 (PTX3), angiopoietin-like protein 3 (ANGPTL3), angiopoietin-like protein 4 (ANGPTL4), angiopoietin-like protein 8 (ANGPTL8) concentrations of children in groups (median)

\begin{tabular}{llcl}
\hline Parameter & Group 1 (NW) & Group 2 (MO) & P \\
\hline PTX3 (pg/ml) median & 2601 & 2403 & NS \\
ANGPTL3 $(\mathrm{pg} / \mathrm{ml})$ median & 1813 & 1667 & NS \\
ANGPTL4 $(\mathrm{ng} / \mathrm{ml})$ median & 15.1 & 17.1 & NS \\
ANGPTL8 $(\mathrm{ng} / \mathrm{ml})$ median & 2.6 & 4.0 & $\leq .001$ \\
\hline
\end{tabular}

Decreasing PTX3 and ANGPTL 3 values as well as increasing ANGPTL4 values were recorded in MO group, however, differences were not statistically significant.

The only parameter, which exhibits significantly increased values in MO children was ANGPTL8/ betatrophin $(p \leq 0.001)$.

In MO children, a correlation between PTX3 and ANGPTL4 was found ( $r=0.297, p \leq 0.05)$. In the same group, a much stronger correlation was observed between ANGPTL3 and HOMA-IR ( $r=$ 0.396, $p \leq 0.01$ ) (Fig.1).

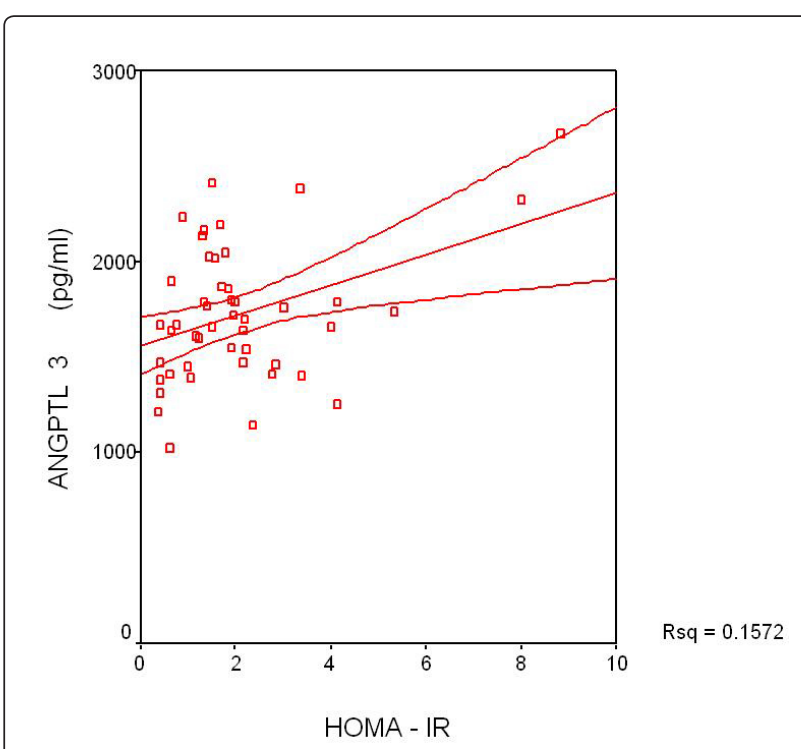

Fig. 1 Correlation between angiopoietin-like protein 3 and homeostatic model assessment of IR in MO group.
Controlling for waist circumference, systolic and diastolic blood pressures, fasting blood glucose, fasting blood insulin and HDL-Chol levels, positive correlations between ANGPTL3 and ANGPTL8/ betatrophin ( $r=0.327, p \leq 0.05)$ as well as PTX3 and ANGPTL4 $(r=0.337, p \leq 0.05)$ were calculated in $\mathrm{MO}$ group (Fig. 2, Fig. 3).

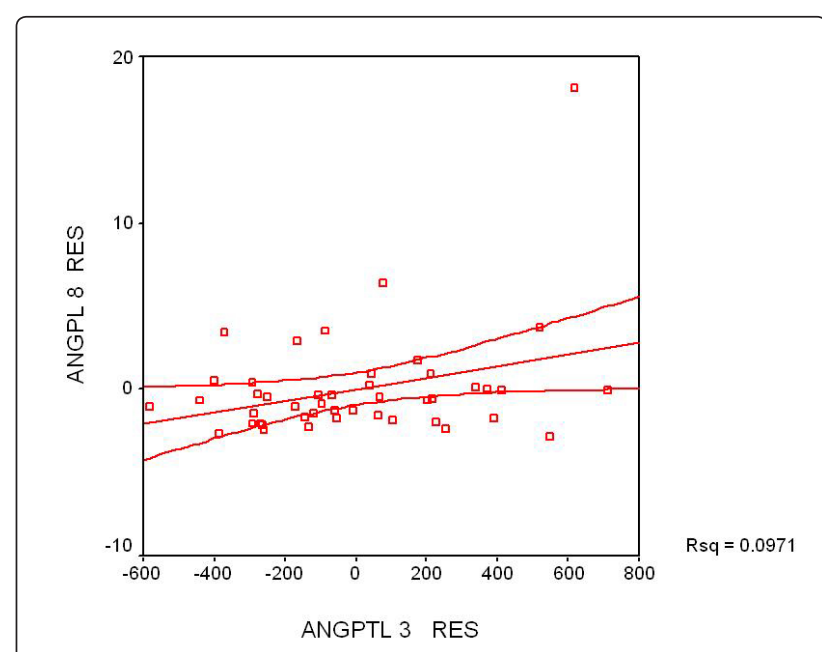

Fig. 2 Partial correlation between angiopoietinlike protein 8 and angiopoietin-like protein 3 in MO group.

None of these correlations were observed in NW group. In both NW and MO groups, when partial correlations were performed by replacing HDL-Chol with TRG, no correlations were detected. 


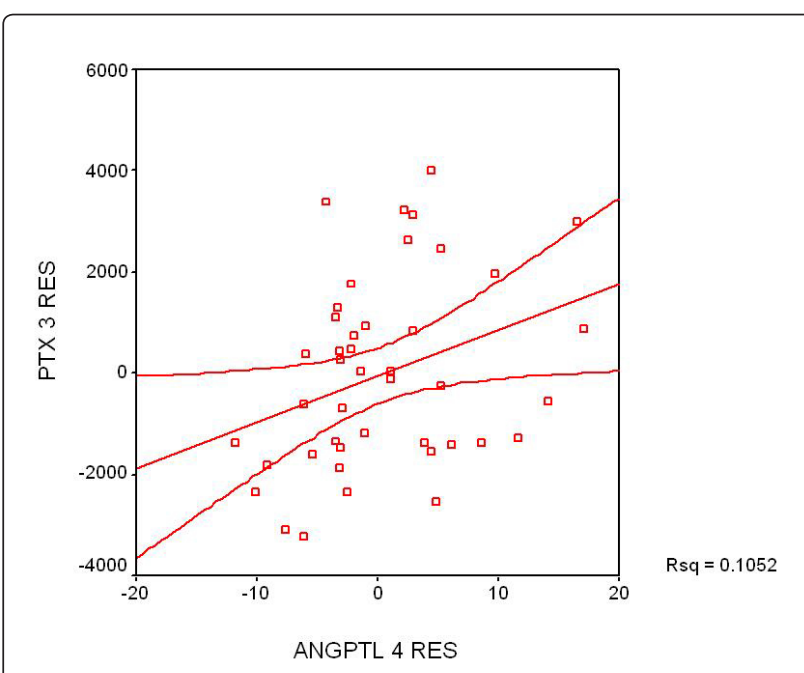

Fig. 3 Partial correlation between pentraxin-3 and angiopoietin-like protein 4 in MO group.

\section{DISCUSSION}

The association of obesity with critical chronic diseases including CVD is well-confirmed. PTX3 may act as a cardioprotective biomarker by decreasing inflammation in cardiovascular bed ${ }^{[2,}$ ${ }^{28]}$. However, it may also contribute to atherogenic process. These contradictory findings make PTX3 an important parameter to be investigated. Negative correlations between PTX3 and IR as well as TRG were reported in some studies ${ }^{[3-5,29]}$ however, such a relation couldn't be found in the others ${ }^{[4,9]}$ including our study.

There are reports explaining that PTX 3 was reduced during obesity ${ }^{[30-32]}$. Our results agree with the results obtained in these studies. Plasma PTX3 showed a nonsignificant trend towards lower levels in $\mathrm{MO}$ group as in one of the above studies ${ }^{[30]}$.

No correlations were found between this parameter and IR, most probably because our study population is composed of prepubertal children. PTX3 can not be described as a cardiovascular biomarker in this period.

While ANGPTL4 plays antilipotoxic role, it is also associated with inflammatory alterations and involved in human atherosclerosis. Increased ANGPTL4 levels were measured in patients with MetS and type 2 diabetes mellitus (T2DM) ${ }^{[18,33-36]}$. In our study, ANGPTL4 levels did not differ between groups. It exhibits a pattern similar to that of PTX3. However, correlation analyses have shown that a hidden relation exists between ANGPTL4 and PTX3 in the very early stages of life.

Our study is the first childhood case-control study in this field. One of the remarkable findings was the detection of the correlation between PTX3 and ANGPTL4 in MO group ( $r=0.297, p \leq 0.05$ ). Controlling for waist circumference, systolic blood pressure, diastolic blood pressure, fasting blood glucose, insulin and HDL-Chol also resulted in even a more strengthened correlation $(r=0.337, p \leq$ 0.05). Considering studies reporting that ANGPTL4 represses foam cell formation and is protective against lipid accumulation to reduce atherosclerosis $[18,33]$, this consistent correlation between PTX3 and ANGPTL4 gives support to studies defending that PTX3 is also a cardioprotective parameter.

ANGPTL3 and ANGPTL4 are molecules with common features. ANGPTL3 is closely associated with arterial wall thickness, possibly it may have a potential role in atherosclerosis pathogenesis ${ }^{[37,38]}$. In our study, concentrations of ANGPTL3 as well as ANGPTL4 did not differ between NW and MO groups as in the case of PTX3. These findings may be due to the characteristic features of the prepubertal study population.

In a recent study performed on a population, which is composed of both overweight and NW children, a significant association was noted between ANGPTL3 levels and HOMA-IR ${ }^{[39]}$. In our study, a positive strong correlation was noted between ANGPTL3 and HOMA-IR ( $r=0.396 ; p \leq 0.01)$ in $\mathrm{MO}$ children. This correlation was not observed in children with normal BMI. This finding is important from this point of view. ANGPTL3 might act as a potential biomarker of IR in MO children. This data supports the reports emphasizing the role of ANGPTL3 in atherosclerosis.

Conflicting results reported for ANGPTL8/ betatrophin concentrations have led to some controversy. In obese children and adolescents, ANGPTL8/betatrophin levels were reported as lower or did not differ compared to those observed in lean children ${ }^{[40-42]}$

In adults, ANGPTL8/betatrophin was introduced as an emerging potential player in dyslipidemia with strong association with HDL-Chol and a potential therapeutic tool for the treatment of dyslipidemia ${ }^{[43]}$. Significantly increased ANGPTL8/betatrophin levels were reported in T2DM with CVDs, T1DM, gestational DM, obesity and MetS ${ }^{[15,44-49]}$. In an experimental study, ANGPTL8/betatrophin overexpression led to dramatically increased serum TRG levels ${ }^{[50]}$. It is also reported that overexpressed ANGPTL8/betatrophin increased serum TRG levels 
in an ANGPTL3-dependent manner ${ }^{[20]}$. These results agree with our findings. In MO group, we have found significantly increased concentrations for this parameter $(p \leq 0.001)$. This profile emphasized the fact that ANGPTL8/betatrophin is an important parameter because it exhibits an increase starting from the early periods of life in MO group.

Adjusting values for MetS parameters, partial correlation analysis emphasized a correlation between ANGPTL3 and ANGPTL8/betatrophin. This supports the notion that ANGPTL8/betatrophin and ANGPTL3 function in the same pathway, that is, ANGPTL8/betatrophin inhibits LPL in an ANGPTL3-dependent manner ${ }^{[51]}$. This finding may be the indicator of ANGPTL8/betatrophin's role in promoting cleavage of ANGPTL3. It may also point out that such correlation emphasizes the importance of ANGPTL8/betatrophin in MO children as a prediagnostic parameter for MetS. This suggests that ANGPTL8/betatrophin may be a therapeutic target for lowering serum TRG levels and may be useful in predicting newly-onset MetS and its progression in clinical settings in adults ${ }^{[52,53]}$.

Considering the limitations of this study, we have kept many parameters (e.g. age, femaleto-male ratio, pubertal status, ethnic origin, living area, nutritional habits, socioeconomic status of the families, medications, and sleep durations) under control for the study groups, however, still some unidentified factors that may influence study parameters other than the above may exist.

It is well-known that these three ANGPTL work in a collaborative manner however, the exact mechanisms are not clearly defined yet ${ }^{[16]}$. In a study, ANGPTL8/betatrophin knock-out mice did not exhibit reduced levels of the ANGPTL3 N-terminal domain ${ }^{[54]}$. Another study reported that in mice with ANGPTL8/betatrophin overexpression, circulating ANGPTL3 levels were reduced ${ }^{[20]}$. The results of our study are consistent with the recently proposed ANGPTL3-4-8 model ${ }^{[51]}$. In this model, ANGPTL3 and ANGPTL8/betatrophin form a complex translocated into the capillaries to inhibit LPL, resulting in lowered levels of circulating ANGPTL3.

Common features of ANGPTL 3 and ANGPTL4, their potential roles in atherosclerosis pathophysiology, cleavage of ANGPTL3 through ANGPTL8/betatrophin have shown that these proteins work in a coordinated manner in the regulation of lipid metabolism. Controlling for MetS parameters including HDL-Chol in $\mathrm{MO}$ group, correlations observed between ANGPTL3 and ANGPTL8/betatrophin as well as PTX3 and ANGPTL4 could not be detected when HDL-Chol was replaced by TRG. This showed that upon evaluation of dyslipidemia in children with MetS, HDL-Chol concentrations are more valuable than TRG concentrations. This study is the first from the evaluation of these four parameters together in $\mathrm{MO}$ children in comparison with NW children point of view. This study provides a basis for further future studies, which will be performed on the matter.

\section{FUNDING}

This study was supported by Namik Kemal University Scientific Research Fund Projects Coordination Unit Project No: NKUBAP.00.20.TU.14.01.

\section{CONFLICTS OF INTERET}

The authors declare that they have no conflict of interest.

\section{LIST OF ABBREVIATIONS}

ANGPTL: Angiopoietin-like proteins

BMI: Body mass index

CRP: C-reactive protein

CVD: Cardiovascular diseases

DP: Diastolic pressure

ELISA: Enzyme-linked immunosorbent assay

HDL-Chol: High density lipoprotein cholesterol

HOMA-IR: Homeostatic model assessment for insulin resistance

IR: Insulin resistance

LDL-Chol: Low density lipoprotein cholesterol

LPL: Lipoprotein lipase

MetS: Metabolic syndrome

MO: Morbid obese

NW: Normal weight

PTX3: Pentraxin 3

SP: Systolic pressure

SPSS: Statistical package for social sciences

TChol: Total cholesterol

TRG: Triacylglycerol 


\section{REFERENCES}

1. Frühbeck G, Toplak H, Woodward E, Yumuk V, Maislos M, Oppert JM; Executive Committee of the European Association for the Study of Obesity. Obesity: the gateway to ill health - an EASO position statement on a rising public health, clinical and scientific challenge in Europe. Obes Facts. 2013; 6: 117-120.

2. Inoue K, Kodama T, Daida H. Pentraxin 3: A novel biomarker for inflammatory cardiovascular disease. Int J Vasc Med. 2012; 2012: 657025.

3. Ogawa T, Kawano Y, Imamura T, Kawakita K, Sagara M, Matsuo T, Kakitsubata Y, Ishikawa T, Kitamura K, Hatakeyama K, Asada Y, Kodama T. Reciprocal contribution of pentraxin 3 and C-reactive protein to obesity and metabolic syndrome. Obesity. 2010; 18: 1871-1874.

4. Kardas F, Akın L, Kurtoglu S, Kendirci M, Kardas Z. Plasma pentraxin 3 as a biomarker of metabolic syndrome. Indian J Pediatr. 2015; 82: 35-38.

5. Chu SH, ParkJH, Lee MK, Jekal Y, Ahn KY, Chung JY, Lee DH, Kim ES, Naruse M, Im JA, Kong D, Chung $\mathrm{CH}$, Lee JW, Chung KM, Kim YB, Jeon JY. The assocation between pentraxin 3 and insulin resistance in obese children at baseline and after physical activity intervention. Clin Chim Acta. 2012; 413: 1430-1437.

6. Bonacina F, Baragetti A, Catapano AL, Norata GD. Long pentraxin 3: experimental and clinical relevance in cardiovascular diseases. Med Inflam. 2013; 2013: 1-10.

7. Suzuki S, Takeishi Y, Niizeki T, Koyama Y, Kitahara T, Sasaki T, Sagara M, Kubota I. Pentraxin 3, a new marker for vascular inflammation, predicts adverse clinical outcomes in patients with heart failure. Am Heart J. 2008; 155: 75-81.

8. Norata GD, Marchesi P, Pulakazhi Venu VK, Pasqualini F, Anselmo A, Moalli F, Pizzitola I, Garlanda C, Mantovani A, Catapano AL. Deficiency of the long pentraxin PTX3 promotes vascular inflammation and atherosclerosis. Circulation. 2009; 120: 699-708.

9. Zanetti M, Bosutti A, Ferreira C, Vinci P, Biolo G, Fonda M, Valente M, Cattin L, Guarnieri G, Barazzoni R. Circulating pentraxin 3 levels are higher in metabolic syndrome with subclinical atherosclerosis: evidence for association with atherogenic lipid profile. Clin Exp Med. 2009; 9: 243-248.

10. Robciuc MR, Maranghi M, Lahikainen A, Rader
D, Bensadoun A, Öörni K, Metso J, Minicocci I, Ciociola E, Ceci F, Montali A, Arca M, Ehnholm C, Jauhiainen M. Angptnl3 deficiency is associated with increased insulin sensitivity, lipoprotein lipase activity, and decreased serum free fatty acids. Atheroscler Thromb Vasc Biol. 2013; 33: 1706-1713.

11. Arca M, Minicocci I, Maranghi M. The angiopoietin-like protein 3: a hepatokine with expanding role in metabolism. Curr Opin. 2013; 24: 313-320.

12. Santulli G. Angiopoietin-like proteins: a comprehensive look. Front Endocrinol. 2014; 5: 1-6.

13. Gómez-Ambrosi J, Pascual E, Catalán V, Rodríguez A, Ramírez B, Silva C, Gil MJ, Salvador J, Frühbeck G. Circulating betatrophin concentrations are decreased in human obesity and type 2 diabetes. J Clin Endocrinol Metab. 2014; 99: E2004-2009.

14. Kersten S. Physiological regulation of lipoprotein lipase. Biochim Biophys Acta. 2014; 1841: 919933.

15. Fu Z, Berhane F, Fite A, Seyoum B, AbouSamra AB, Zhang R. Elevated circulating lipasin/ ANGPTL8/betatrophin in human type 2 diabetes and obesity. Sci Rep. 2014; 4: 1-5.

16. Zhang $R$, Abou-Samra AB. A dual role of lipasin (ANGPTL8/betatrophin in lipid metabolism and glucose homeostasis: consensus and controversy. Cardiovasc Diabetol. 2014; 13: 1-9.

17. Li Y, Teng C. Angiopoietin-like proteins 3, 4 and 8: regulating lipid metabolism and providing new hope for metabolic syndrome. J Drug Target. 2014; 22: 679-687.

18. Georgiadi A, Wang Y, Stienstra R, Tjeerdema N, Janssen A, Stalenhoef $A$, van der Vliet JA, de Roos A, Tamsma JT, Smit JW, Tan NS, Müller M, Rensen PC, Kersten S. Overexpression of angiopoietin-like protein 4 protects against atherosclerosis development. Atheroscler Thromb Vasc Biol. 2013; 33: 1529-1537.

19. Li Y, He PP, Zhang DW, Zheng XL, Cayabyab FS, Yin WD, Tang CK. Lipoprotein lipase: From gene to atherosclerosis. Atherosclerosis. 2014; 237: 597-608.

20. Quagliarini F, Wang Y, Kozlitina J, Grishin NV, Hyde R, Boerwinkle E, Valenzuela DM, Murphy AJ, Cohen JC, Hobbs HH. Atypical angiopoietinlike protein that regulates ANGPTL3. Proc Nat Acad Sci USA. 2012; 109: 19751-19756.

21. Guo T, Huang L, Liu C, Shan S, Li Q, Ke L, 
Cheng $B$. The clinical value of inflammatory biomarkers in coronary artery disease: PTX3 as a new inflammatory marker. Exp Gerontol. 2017; 97: 64-67.

22. Casula M, Montecucco F, Bonaventura A, Liberale L, Vecchié A, Dallegri F, Carbone $F$. Update on the role of Pentraxin 3 in atherosclerosis and cardiovascular diseases. Vascul Pharmacol. 2017; pii: S1537. 1891(17)30181-7.

23. Kersten S. Angiopoietin-like 3 in lipoprotein metabolism. Nat Rev Endocrinol. 2017; 13(12): 731-739.

24. Graham MJ, Lee RG, Brandt TA, Tai LJ, Fu W, Peralta R, Yu R, Hurh E, Paz E, McEvoy BW, Baker BF, Pham NC, Digenio A, Hughes SG, Geary RS, Witztum JL, Crooke RM, Tsimikas S. Cardiovascular and metabolic effects of ANGPTL3 antisense oligonucleotides. N Engl J Med. 2017; 377(3): 222-232.

25. Ye Q, Tian GP, Cheng HP, Zhang X, Ou X, Yu $X H$, Tan $R Q$, Yang $F Y$, Gong D, Huang $C$, Pan YJ, Zhang J, Chen LY, Zhao ZW, Xie W, Li L, Zhang M, Xia XD, Zheng XL, Tang CK. MicroRNA-134 promotes the development of atherosclerosis via the ANGPTL4/LPL pathway in apolipoprotein E knockout mice. J Atheroscler Thromb. 2017; doi: 10.5551/jat.40212.

26. He XW, Shen YG, Zhu M, Hu XF, Zheng Z, Liu P, Li C, Zhu F, Jin XP. Angiopoietin-like protein 4 serum levels and gene polymorphisms are associated with large artery atherosclerotic stroke. J Neurol Sci. 2016; 362: 333-338.

27. Xu L, Guo ZN, Yang Y, Xu J, Burchell SR, Tang J, Zhang J, Xu J, Zhang JH. Angiopoietin-like 4: A double-edged sword in atherosclerosis and ischemic stroke? Exp Neurol. 2015; 272: 61-66.

28. Bonacina F, Barbieri SS, Amadio P, Doni A, Sironi M, Bottazzi B, Garlanda C, Tremoli E, Mantovani A, Catapano AL, Norata GD. Pentraxin 3 deficiency is associated with increased arterial thrombosis in animal models. Atherosclerosis. 2016; 252: e250-e251

29. Yamasaki K, Kurimura M, Kasai T, Sagara $M$, Kodama T, Inoue K. Determination of physiological plasma pentraxin 3 (PTX3) levels in healthy populations. Clin Chem Lab Med. 2009; 47: 471-477.

30. Osorio-Conles O, Guitart M, Chacón MR, Maymo-Masip E, Moreno-Navarrete JM, Montori-Grau M, Näf S, Fernandez-Real JM, Vendrell J, Gómez-Foix AM. Plasma PTX3 protein levels inversely correlate with insulin secretion and obesity, whereas visceral adipose tissue PTX3 gene expression is increased in obesity. Am J Physiol Endocrinol Metab. 2011; 301: E1254-1261.

31. Slusher AL, Mock JT, Whitehurst M, Maharaj A, Huang CJ. The impact of obesity on pentraxin 3 and inflammatory milieu to acute aerobic exercise. Metabolism. 2015; 64: 323-329.

32. Slusher AL, Huang CJ. Association of pentraxin 3 with insulin resistance and glucose response following maximal aerobic exercise in obese and normal-mass individuals. Can J Physiol Pharmacol. 2016; 94: 734-738.

33. Aryal B, Rotllan N, Araldi E, Ramirez CM, He S, Chousterman BG, Fenn AM, Wanschel A, Madrigal-Matute $\mathrm{J}$, Warrier $\mathrm{N}$, Martin-Ventura JL, Swirski FK, Suarez Y, Fernandez-Hernando C. ANGPTL4 deficiency in haematopoietic cells promotes monocyte expansion and atherosclerosis progression. Nat Commun. 2016; 7: 12313.

34. Guo L, Li SY, Ji FY, Zhao YF, Zhong Y, Lv XJ, Wu XL, Qian GS. Role of Angptl4 in vascular permeability and inflammation. Inflam Res. 2014; 63: 13-22.

35. Tjeerdema N, Georgiadi A, Jonker JT, van Glabbeek M, Alizadeh Dehnavi R, Tamsma JT, Smit JW, Kersten S, Rensen PC. Inflammation increases plasma angiopoietin-like protein 4 in patients with the metabolic syndrome and type 2 diabetes. BMJ Open Diabetes Res Care. 2014; 2: e000034.

36. Katano H, Yamada K. Upregulation of ANGPTL4 messenger RNA and protein in severely calcified carotid plaques. J Stroke Cerebrovasc Dis. 2014; 23: 933-947.

37. Mehta N, Qamar A, Qu L, Qasim AN, Mehta NN, Reilly MP, Rader DJ. Differential association of plasma angiopoietin-like proteins 3 and 4 with Ipid and metabolic traits. Atheroscler Thromb Vasc Biol. 2014; 34: 1057-1063.

38. Hatsuda S, Shoji T, Shinohara K, Kimoto E, Mori $\mathrm{K}$, Fukumoto S, Koyama H, Emoto M, Nishizawa Y. Association between plasma angiopoietin-like protein 3 and arterial wall thickness in healthy subjects. J Vasc Res. 2007; 44: 61-66.

39. Chung HS, Lee MJ, Hwang SY, Lee HJ, Yoo HJ, Seo JA, Kim SG, Kim NH, Baik SH, Choi DS, Kim SM, Choi KM. Circulating angiopoietinlike protein 8 (ANGPTL8) and ANGPTL3 concentrations in relation to anthropometric and metabolic profiles in Korean children: a prospective cohort study. Cardiovasc Diabetol. 
2016; 15: 1.

40. Tuhan $H, A b a c ı A$, Anık A, Çatlı G, Küme T, Çalan ÖG, Acar S, Böber E. Circulating betatrophin concentration is negatively correlated with insulin resistance in obese children and adolescents. Diabetes Res Clin Pract. 2016; 114: 37-42.

41. Wu S, Gao H, Ma Y, Fu L, Zhang C, Luo X. Characterisation of betatrophin concentrations in childhood and adolescent obesity and insulin resistance. Pediatr Diabetes. 2016; 17: 53-60.

42. Roth CL, Elfers C, Lass N, Reinehr T. Betatrophin: no relation to glucose metabolism or weight status in obese children before and after lifestyle intervention. Pediatr Diabetes. 2017; 18(6): 485-491.

43. Gomez-Ambrosi J, Pascual-Corrales E, Catalan V, Rodriguez A, Ramirez B, Romero S, Vila N, Ibanez P, Margall MA, Silva C, Gil MJ, Salvador J, Frühbeck G. Altered concentrations in dyslipidemia evidence a role for ANGPTL8/ ANGPTL8/betatrophin in lipid metabolism in humans. J Clin Endocr Metab. 2016; 101: 38033811.

44. Huang $Y$, Fang $\mathrm{C}$, Guo H, Hu J. Increased angiopoietin-like protein 8 levels in patients with type 2 diabetes and cardiovascular disease. Diabetes Res Clin Pract. 2016; 120: 229-231.

45. Kong FJ, Ma LL, Li G, Chen YX, Zhou JQ. Circulating ANGPTL8/betatrophin levels and gestational diabetes mellitus: A systematic review and meta-analysis. PloS One. 2017; 12: e0169941.

46. Abu-Farha M, Al-Khairi I, Cherian P, Chandy B, Sriraman D, Alhubail A, Al-Refaei F, AlTerki A, Abubaker J. Increased ANGPTL3, 4 and ANGPTL8/ANGPTL8/betatrophin expression levels in obesity and T2D. Lipids Health Dis. 2016; 15: 181.
47. Espes D, Lau J, Carlsson PO. Increased circulating levels of ANGPTL8/betatrophin in individuals with long-standing type 1 diabetes. Diabetologia. 2014; 57: 50-53.

48. Hu H, Sun W, Yu S, Hong X, Qian W, Tang B, Wang D, Yang L, Wang J, Mao C, Zhou L, Yuan G. Increased circulating levels of ANGPTL8/ betatrophin in newly diagnosed type 2 diabetic patients. Diabetes Care. 2014; 37: 2718-2722.

49. Abu-Farha M, Abubaker J, Al-Khairi I, Cherian P, Noronha F, Kavalakatt S, Khadir A, Behbehani K, Alarouj M, Bennakhi A, Elkum N. Circulating angiopoietin-like protein (ANGPTL8/betatrophin) association with HsCRP and metabolic syndrome. Cardiovasc Diabetol. 2016; 15: 25.

50. Zhang R. Lipasin, a novel nutritionally-regulated liver-enriched factor that regulates serum triglyceride levels. Biochem Biophys Res Commun. 2012; 424: 786-792.

51. Zhang R. The ANPTL3-4-8 model, a molecular mechanism for triglyceride trafficking. Open Biol. 2016; 6: 150272.

52. Fu Z, Abou-Samra AB, Zhang R. A lipasin/ Angptl8 monoclonal antibody lowers mouse serum triglycerides involving increased postprandial activity of the cardiac lipoprotein lipase. Sci Rep. 2015; 5: 18502.

53. Wang $\mathrm{H}$, Lai $\mathrm{Y}$, Han $\mathrm{C}$, Liu $\mathrm{A}$, Fan $\mathrm{C}$, Wang $\mathrm{H}$, Zhang H, Ding S, Teng W, Shan Z. The effects of serum ANGPTL8/ANGPTL8/betatrophin on the risk of developing the metabolic syndrome-A prospective study. Sci Rep. 2016; 6: 28431.

54. Wang Y, Quagliarini F, Gusarova V, Gromada J, Valenzuela DM, Cohen JC, Hobbs HH. Mice lacking ANGPTL8 (ANGPTL8/betatrophin) manifest disrupted triglyceride metabolism without impaired glucose homeostasis. Proc Nat Acad Sci USA. 2013; 110: 16109-16114. 